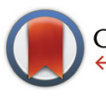

CrossMark

\&click for updates

Cite this: Dalton Trans., 2016, 45

3627

Received 16th November 2015,

Accepted 19th January 2016

DOI: $10.1039 / c 5 d t 04506 a$

www.rsc.org/dalton

\title{
Cationic ruthenium alkylidene catalysts bearing phosphine ligands $\dagger$
}

\author{
Koji Endo and Robert H. Grubbs*
}

\begin{abstract}
The discovery of highly active catalysts and the success of ionic liquid immobilized systems have accelerated attention to a new class of cationic metathesis catalysts. We herein report the facile syntheses of cationic ruthenium catalysts bearing bulky phosphine ligands. Simple ligand exchange using silver(1) salts of non-coordinating or weakly coordinating anions provided either $\mathrm{PPh}_{3}$ or chelating $\mathrm{Ph}_{2} \mathrm{P}\left(\mathrm{CH}_{2}\right)_{n} \mathrm{PPh}_{2}(n=2$ or 3) ligated cationic catalysts. The structures of these newly reported catalysts feature unique geometries caused by ligation of the bulky phosphine ligands. Their activities and selectivities in standard metathesis reactions were also investigated. These cationic ruthenium alkylidene catalysts reported here showed moderate activity and very similar stereoselectivity when compared to the second generation ruthenium dichloride catalyst in ring-closing metathesis, cross metathesis, and ring-opening metathesis polymerization assays.
\end{abstract}

\section{Introduction}

Olefin metathesis is a convenient and powerful methodology for the construction of carbon-carbon double bonds. ${ }^{1}$ Due to their high activity and functional group tolerance, rutheniumbased catalysts have been utilized in a variety fields including natural product synthesis, ${ }^{2}$ biochemistry, ${ }^{3}$ green chemistry ${ }^{4}$ and polymer chemistry. ${ }^{5}$ Along with the expansion of these applications, the catalysts themselves have dramatically evolved. In particular, the introduction of an $N$-heterocyclic carbene (NHC) ligand in place of a phosphine ligand led to a large enhancement in catalyst activity and stability. ${ }^{6}$ These types of catalysts are widely used in both academic and industrial laboratories.

The modification of X-type ligands, which are generally chlorides in the original catalyst systems, is becoming a popular avenue of investigation because of their easy substitution and large impact on catalyst performance. Buchmeiser et al. first utilized silver(I) salts to remove a chloride ligand and simultaneously introduce an anionic ligand in its place. ${ }^{7}$ They expanded this methodology to form solid supported systems where a catalyst is bound to a monolith through a polymeric X-type ligand. ${ }^{7 a, b}$ Additionally, Hoveyda et al. formed a bidentate chiral NHC ligated complex through similar ligand

Arnold and Mabel Beckman Laboratory of Chemical Synthesis, Division of Chemistry and Chemical Engineering, California Institute of Technology, Pasadena, California 91125, USA.E-mail: rhg@caltech.edu

$\dagger$ Electronic supplementary information (ESI) available: NMR spectra and metathesis data. CCDC 784488. For ESI and crystallographic data in CIF or other electronic format see DOI: $10.1039 / \mathrm{c} 5 \mathrm{dt} 04506 \mathrm{a}$ exchange reactions which enabled highly enantioselective asymmetric ring-opening cross metathesis (AROM) and cross metathesis $(\mathrm{CM}){ }^{8}$ We recently reported a family of NHC chelated catalysts which were produced by coordination of two pivalate ligands and subsequent intramolecular $\mathrm{C}-\mathrm{H}$ bond activation. ${ }^{9}$ This family of catalysts has showed very high activity and $Z$-selectivity in a variety of metathesis reactions. ${ }^{10}$

The formation of catalysts that are cationic at the metal center or contain pendant positively charged groups has been previously reported (1-6 in Fig. 1). One of the remarkable examples are ionic liquid tagged catalysts, like 1 and $2,{ }^{11}$ that have an oligomeric tether capped with a cationic imidazolium group. Due to their cationic charge, they can be immobilized in an ionic liquid phase and behave as supported catalysts, achieving efficient catalyst recyclability. Several cationic catalysts, in which the ruthenium center is positively charged, have been also reported. ${ }^{12}$ Among these catalysts, 3 shows much higher activity in ring-opening metathesis polymerization (ROMP) reactions of cyclooctene compared to standard NHC-ligated catalyst. ${ }^{12 a}$ In ring-closing metathesis (RCM) reactions of a tetra-substituted olefin, which is one of the toughest olefin metathesis transformations, 5 surpasses the second generation catalyst. ${ }^{12 c}$

Relying on the recent successes mentioned above, modification of the X-type ligand to produce cationic rutheniumbased catalysts is highly promising in improving catalyst efficiency and expanding their applications. Therefore, the discovery of facile methodologies to prepare these types of catalysts is highly desired. Here we report the convenient syntheses of cationic catalysts bearing bulky phosphine ligands, and explore their reactivity in standard metathesis reactions. 


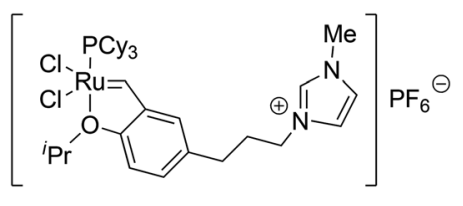

1

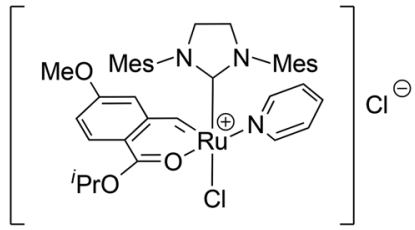

4

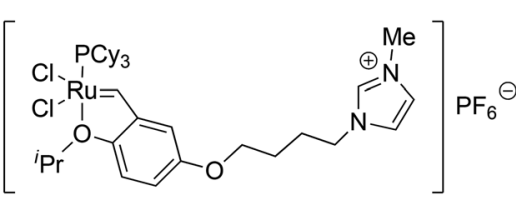

2

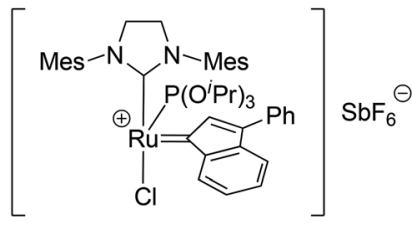

5

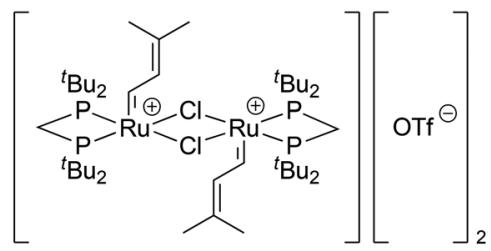

3

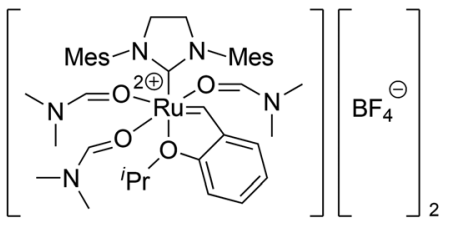

6

Fig. 1 Examples of cationic ruthenium alkylidene catalysts.

\section{Results and discussion}

\section{Catalyst syntheses}

We chose $\left[\mathrm{H}_{2} \mathrm{IMes}_{2}\right] \mathrm{RuCl}_{2}\left[=\mathrm{CH}-\mathrm{o}-\left(\mathrm{O}^{\mathrm{i}} \mathrm{Pr}\right) \mathrm{C}_{6} \mathrm{H}_{4}\right]\left(7, \mathrm{H}_{2} \mathrm{I}=\right.$ imidazolidinylidene, $\mathrm{Mes}=$ mesityl) as a starting complex due to its high activity in various metathesis reactions. ${ }^{13}$ It was previously reported that $\mathrm{AgBF}_{4}$ is capable of extracting a single chloride ligand from complex $7 .{ }^{12 d}$ When 7 was reacted with $\mathrm{AgBF}_{4}$ in the presence of $\mathrm{PPh}_{3}$, a cationic catalyst bearing a bulky $\mathrm{PPh}_{3}$ ligand (8a) was obtained in high yield. In the same manner, other silver salts of non-coordinating or weekly coordinating anions also provided similar cationic catalysts $(\mathbf{8 b}-\mathbf{d})$ (Scheme 1). In the ${ }^{1} \mathrm{H}$ NMR spectra of $\mathbf{8}$, signals for the methyl groups of the two $\mathrm{N}$-mesityl substituents appear as all inequivalent, suggesting that the bulky $\mathrm{PPh}_{3}$ ligand hinders rotation of the NHC ligand at room temperature. X-ray quality crystals of 8c were grown and the crystal structure indicates steric repulsion between a phenyl group of the $\mathrm{PPh}_{3}$ ligand and the $\mathrm{N}$-mesityl group of the NHC ligand (Fig. 2). Compared to $7,{ }^{13}$ 8c has smaller C1-Ru1-O1 angle $\left(96.73(5)^{\circ}\right.$ versus $\left.176.2(14)^{\circ}\right)$ and larger C1-Ru1-Cl1 angle $\left(154.88(4)^{\circ}\right.$ versus $96.6(12)^{\circ}$ and $\left.90.9(12)^{\circ}\right)$. The latter structural feature is also observed in previously reported complexes $4^{12 b}$ and $5^{12 c}$ (Fig. 1).

Next, we were able to synthesize cationic diphosphine chelated catalysts derived from $\left[\mathrm{H}_{2} \mathrm{IMes}_{2}\right] \mathrm{RuCl}_{2}\left(\mathrm{PPh}_{3}\right)(=\mathrm{CHPh})(\mathbf{9})^{14}$

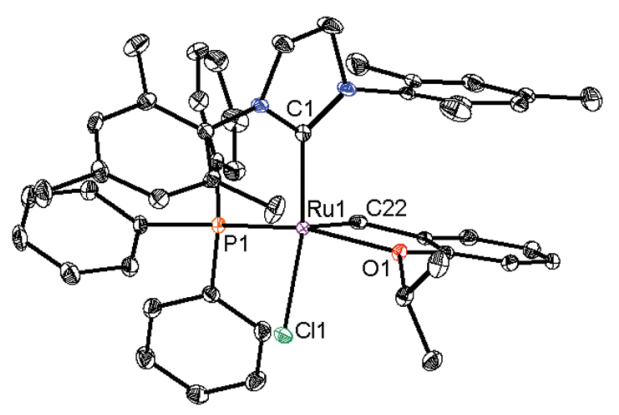

Fig. 2 X-ray crystal structure of 8c. Displacement ellipsoids are drawn at $50 \%$ probability. For clarity, hydrogen atoms and the counter anion $\mathrm{PF}_{6}$ have been omitted. Selected bond length (Å) for 8c: C1-Ru1 2.0723 (15), C22-Ru1 1.8349(15), O1-Ru1 2.3442(10), Cl1-Ru1 2.3517(4), P1Ru1 2.2788(4).

(Scheme 2). First, 9 was exposed to TMS(OTf) causing the substitution of one chloride ligand with an OTf anion, yielding mono-triflate complex 10. Next, diphosphines DPPE and DPPP were added, subsequently replacing both the labile $\mathrm{PPh}_{3}$ and OTf ligands, and afforded cationic diphosphine chelated complexes $\mathbf{1 1 a}$ and $\mathbf{1 1 b}$, respectively. As a result of coupling with two unequivalent phosphorus atoms, the signal of the benzylidene proton appeared as doublet of doublets in the ${ }^{1} \mathrm{H}$ NMR
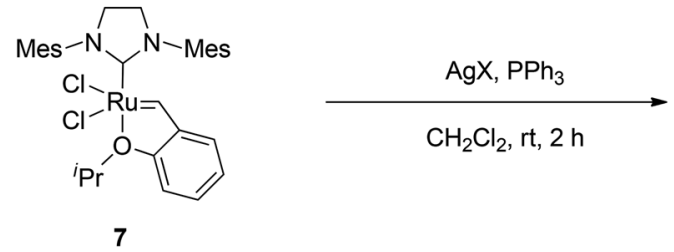

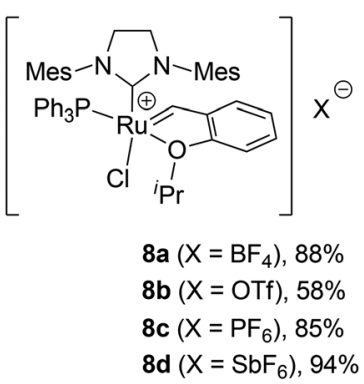

Scheme 1 


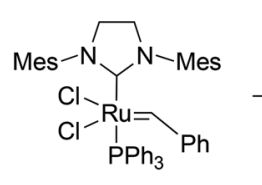

9

1. TMS(OTf), $\mathrm{CH}_{2} \mathrm{Cl}_{2}, \mathrm{rt}, 1 \mathrm{~h}$ 2. DPPE or DPPP, $\mathrm{CH}_{2} \mathrm{Cl}_{2}, \mathrm{rt}, 2 \mathrm{~h}$<smiles>CC1CC1</smiles>

spectrum. Additionally, a large difference in the chemical shifts of the two phosphorus atoms in the ${ }^{31} \mathrm{P}$ NMR spectrum suggests that the diphosphine ligand coordinates to the ruthenium center with one phosphorus atom at the equatorial position and the other one at the axial position in the catalyst.

\section{Metathesis assays}

Ring closing metathesis (RCM). First, the standard RCM reaction of diethyldiallyl malonate $(\mathbf{1 2})^{15}$ was carried out using catalysts $\mathbf{8 a}-\mathbf{c}$ and $\mathbf{1 1 a}-\mathbf{b}$ in order to evaluate their activities (Scheme 3). As shown in Fig. 3, mono-phosphine catalysts 8a-c

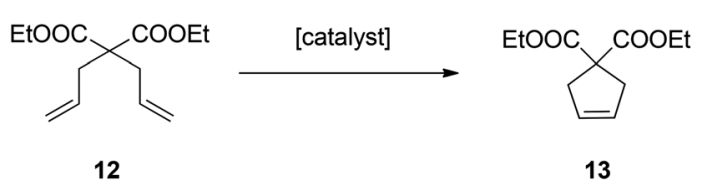

Scheme 3

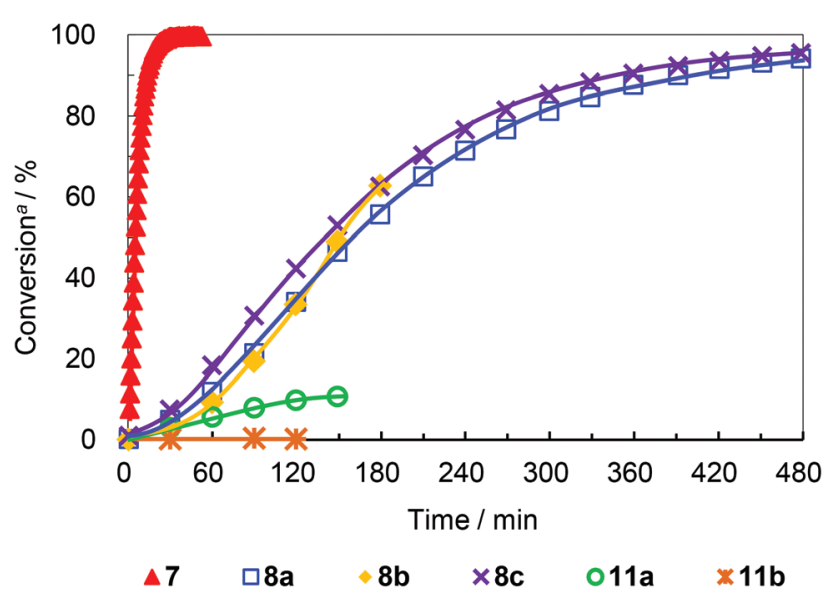

Fig. 3 Plots for conversion vs. time for RCM of 12 . All reactions were carried out using $0.080 \mathrm{mmol}$ of 12 and $0.80 \mu \mathrm{mol}$ of catalyst in $0.8 \mathrm{ml}$ of $\mathrm{CD}_{2} \mathrm{Cl}_{2}$ at $30{ }^{\circ} \mathrm{C}$. Data for 7 is from ref. 15 . ${ }^{a}$ Conversion of 12 to 13 determined by ${ }^{1} \mathrm{H}$ NMR analysis. showed moderate activities and the conversion to reach $\sim 90 \%$ after 6 hours. The lower activity of $\mathrm{PPh}_{3}$-substituted complexes 8a-c compared to 7 may be due to the steric bulkiness of the phosphine ligand that possibly hinders olefin coordination. It should be noted that no significant dependence of the counter anions on metathesis activity was observed. The anions, which potentially could coordinate to the ruthenium center and compete with olefin coordination did not affect catalyst reactivity under the presented conditions. Alternatively, diphosphinesubstituted catalysts 11a-b exhibited poor activity, providing significantly low to no conversion of 12. Considering the mechanism of initiation, it is seemingly necessary to dissociate one phosphorous arm from the ruthenium center in order to make a vacant site for coordination of incoming olefin (Fig. 4). Because the chelated form seems like a dormant species, the high energy barrier of the dechelation of the diphosphine ligand is thought to decelerate the catalyst initiation and overall metathesis reaction.

Cross metathesis (CM). In order to evaluate the activity and stereoselectivity of these new catalysts, the standard CM reaction of allylbenzene (14) and cis-1,4-diacetoxy-2-butene $(\mathbf{1 5})^{\mathbf{1 5}}$ was carried out (Scheme 4). Selected data for the cationic catalysts are summarized in Table 1 and plotted in Fig. 5. Similar to the RCM assay above, while $\mathbf{8 a - b}$ and 11a showed moderate activities, no conversion was observed when $\mathbf{1 1 b}$ was used. It has been reported that some asymmetric ruthenium-based catalysts substituted with one bulky $\mathrm{X}$-type ligand tend to give lower $E / Z$ ratio of the products compared to catalyst $7 .{ }^{16}$ In one case, a ruthenium catalyst bearing a single bulky thiolate ligand (18) provided an $E / Z$ ratio of 0.20 in the homocoupling of substrate 14 (Fig. 6). ${ }^{16 b}$ However, the $E / Z$ ratio of product 16 formed by the cationic catalysts reported here are very similar to the one by the dichloro analogue 7 (Fig. 5(b)). This possibly indicates that the phosphine ligands are too far from the reaction center to influence the stereoselectivity (Fig. 6).

Ring opening metathesis polymerization (ROMP). Next, the ROMP of norbornene (19) was tested with the presented cationic catalysts (Scheme 5). In all cases, an immediate increase in the viscosity of the reaction solution was observed after stirring substrate 19 with the catalysts, indicating rapid 

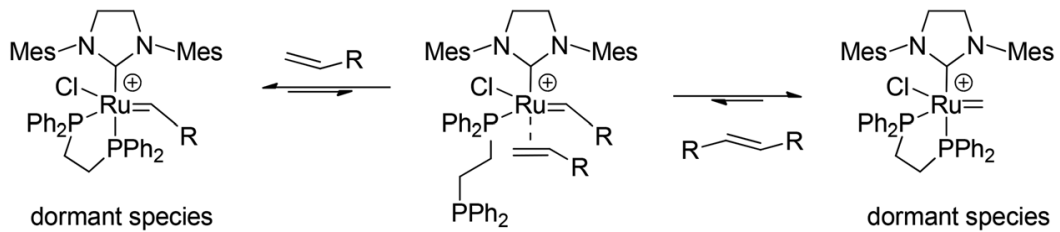

dormant species

Fig. 4 One plausible mechanism of catalyst initiation for 11a.

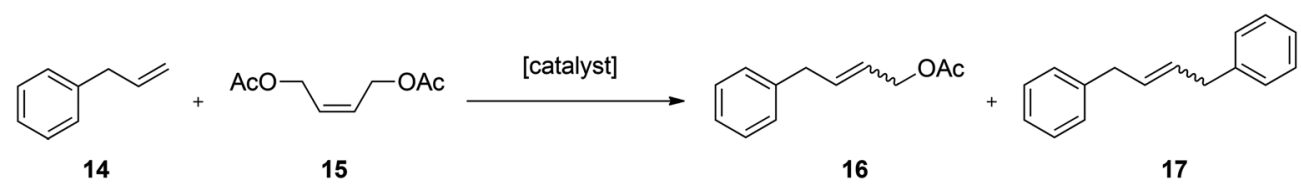

Scheme 4

Table 1 Selected data for the CM of 14 and $15^{a}$

\begin{tabular}{|c|c|c|c|c|c|c|c|c|}
\hline & Cat. & $\begin{array}{l}\text { Cat. load }{ }^{b}, \\
\text { mol\% }\end{array}$ & Solvent & $\begin{array}{l}\text { Time, } \\
\text { min }\end{array}$ & \multicolumn{2}{|l|}{16} & \multicolumn{2}{|l|}{17} \\
\hline $1^{e}$ & 7 & 2.5 & $\mathrm{CH}_{2} \mathrm{Cl}_{2}$ & 30 & 72 & 10.1 & 5.0 & 5.9 \\
\hline \multirow[t]{2}{*}{2} & \multirow[t]{2}{*}{$8 a$} & \multirow[t]{2}{*}{2.5} & \multirow[t]{2}{*}{$\mathrm{CH}_{2} \mathrm{Cl}_{2}$} & 30 & 34 & 3.3 & 0.0 & $(\mathrm{NA})^{f}$ \\
\hline & & & & 120 & 74 & 6.1 & 2.5 & $(\mathrm{NA})^{f}$ \\
\hline 3 & $8 b$ & 2.5 & $\mathrm{CH}_{2} \mathrm{Cl}_{2}$ & 30 & 28 & 3.2 & 0.0 & $(\mathrm{NA})^{f}$ \\
\hline \multirow{3}{*}{5} & & & & 120 & 34 & 3.2 & 0.0 & $(\mathrm{NA})^{f}$ \\
\hline & \multirow[t]{2}{*}{$11 b$} & \multirow[t]{2}{*}{5.0} & \multirow[t]{2}{*}{$\mathrm{CH}_{2} \mathrm{Cl}_{2}$} & 30 & 0.0 & $(\mathrm{NA})^{f}$ & 0.0 & $(\mathrm{NA})^{f}$ \\
\hline & & & & 120 & 0.0 & $(\mathrm{NA})^{f}$ & 0.0 & $(\mathrm{NA})^{f}$ \\
\hline
\end{tabular}

${ }^{a}$ All reactions were carried out using $0.20 \mathrm{mmol}$ of $\mathbf{1 4}, 0.40 \mathrm{mmol}$ of 15 and $0.10 \mathrm{mmol}$ of tridecane (internal standard for GC analysis) in $1.0 \mathrm{ml}$ of solvent at $23{ }^{\circ} \mathrm{C} .{ }^{b}$ Based on $\mathbf{1 4} .{ }^{c}$ Conversion of $\mathbf{1 4}$ to the product determined by GC analysis. ${ }^{d}$ Molar ratio of $E$ isomer and $Z$ isomer of the product determined by GC analysis. ${ }^{e}$ Ref. $15 .{ }^{f} \mathrm{GC}$ signal of the product was too small to quantify.

polymerization. The conversion and $E / Z$ ratio of the product poly-norbornene 20 are summarized in Table $2.8 \mathbf{8 a}-\mathbf{b}$ and 11a were able to complete the reaction within $30 \mathrm{~min}$ at the presented condition. Even 11b, which showed negligible activity in the RCM and CM reactions above, provided $70 \%$ yield of $\mathbf{2 0}$ after $30 \mathrm{~min}$.

\section{Experimental section}

\section{General information}

Atmosphere. All reactions were carried out in dry glassware under an argon atmosphere using standard Schlenk techniques or in a Vacuum Atmospheres Glovebox under a nitrogen atmosphere unless otherwise specified.

Solvents. $\mathrm{CD}_{2} \mathrm{Cl}_{2}$ was dried over $\mathrm{CaH}_{2}$ and vacuum transferred to a dry Schlenk flask and subsequently degassed with argon. Ethyl vinyl ether and isopropyl alcohol were used as received. All the other solvents were purified by passage through solvent purification columns and further degassed with argon. ${ }^{18}$
Materials. $\left[\mathrm{H}_{2} \mathrm{IMes}_{2}\right] \mathrm{RuCl}_{2}\left[=\mathrm{CH}-o-\left(\mathrm{O}^{\mathrm{i}} \mathrm{Pr}\right) \mathrm{C}_{6} \mathrm{H}_{4}\right]\left(7, \mathrm{H}_{2} \mathrm{I}=\right.$ imidazolidinylidene, Mes = mesityl) was obtained from Materia, Inc. $\left[\mathrm{H}_{2} \mathrm{I}(\mathrm{Mes})_{2}\right] \mathrm{RuCl}_{2}\left(\mathrm{PPh}_{3}\right)(=\mathrm{CHPh}) \quad$ (9) was synthesized according to the literature procedure. ${ }^{14}$ Diethyldiallyl malonate (12), allylbenzene (14), cis-1,4-diacetoxy-2-butene (15) and tridecane were distilled over $\mathrm{CaH}_{2}$ and stored under nitrogen in Schlenk flasks. Norbornene (19) was purified by sublimation before use. All the other commercially available reagents were used as received without further purification.

Instruments. ${ }^{1} \mathrm{H},{ }^{13} \mathrm{C}$ and ${ }^{31} \mathrm{P}$ NMR spectra were recorded on a Varian $500 \mathrm{MHz}$ spectrometer or a Varian $300 \mathrm{MHz}$ spectrometer. High-resolution mass spectra were provided by the California Institute of Technology Mass Spectrometry Facility using JEOL JMS-600H High Resolution Mass Spectrometer. $\mathrm{X}$-ray crystallographic data were collected by the California Institute of Technology Beckman Institute X-ray Crystallography Facility using Bruker KAPPA APEXII X-ray diffractometer. Gas chromatography data were obtained using Agilent 6850 FID gas chromatograph equipped with a DB-Wax polyethylene glycol capillary column (Agilent). 
(a)

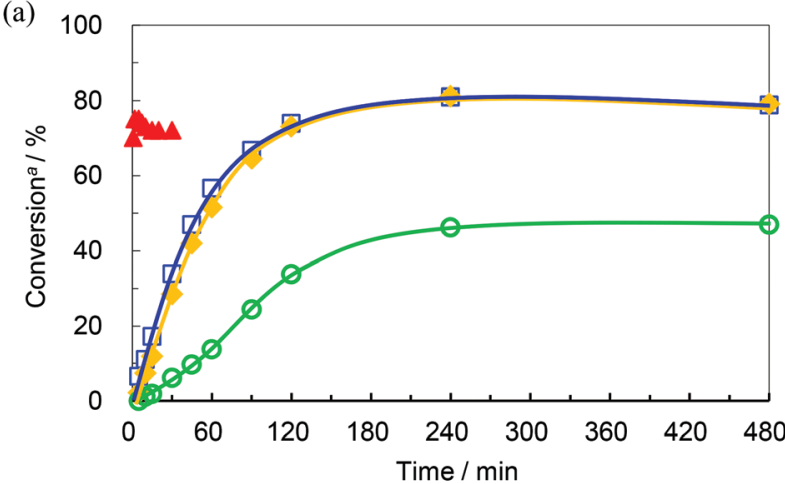

(b)

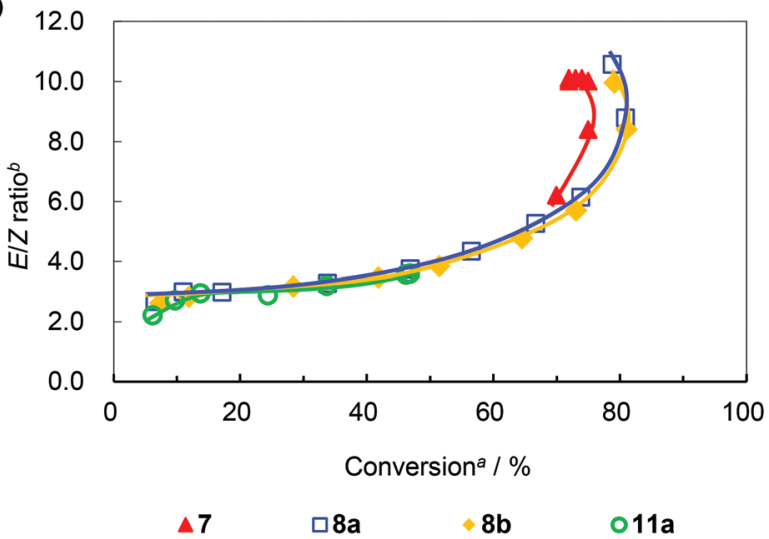

Fig. 5 Plots for (a) conversion vs. time and (b) $E / Z$ ratio vs. conversion for CM of 14 and $15 .{ }^{a}$ Conversion of 14 to 16 determined by GC analysis. ${ }^{17 b}$ Molar ratio of $E$ isomer and $Z$ isomer of 16 determined by GC analysis.

(a)

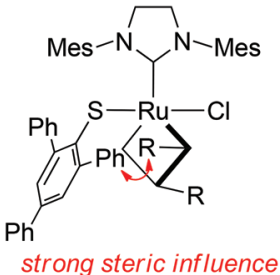

(b)

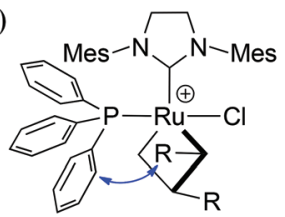

weak steric influence

Fig. 6 Plausible intermediates for (a) 18 and (b) 8
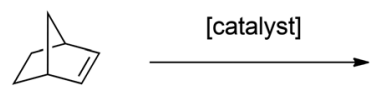

19

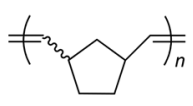

20
Scheme 5
Table 2 Data for the ROMP of $19^{a}$

\begin{tabular}{lllllll}
\hline & & & & & \multicolumn{2}{l}{$\mathbf{2 0}$} \\
\cline { 5 - 7 } Entry & Cat. & $\begin{array}{l}\mathrm{Cat}^{2} \mathrm{moad}^{b}, \\
\text { mol\% }\end{array}$ & Solvent & $\begin{array}{l}\text { Time, } \\
\text { min }\end{array}$ & Conv. ${ }^{c}, \%$ & $E / Z^{d}$ \\
\hline 1 & 7 & 1.0 & $\mathrm{CD}_{2} \mathrm{Cl}_{2}$ & 30 & 100 & 0.69 \\
2 & $\mathbf{8 a}$ & 1.0 & $\mathrm{CD}_{2} \mathrm{Cl}_{2}$ & 30 & 100 & 0.70 \\
3 & $\mathbf{8 b}$ & 1.0 & $\mathrm{CD}_{2} \mathrm{Cl}_{2}$ & 30 & 100 & 0.69 \\
4 & $\mathbf{1 1 a}$ & 1.0 & $\mathrm{CD}_{2} \mathrm{Cl}_{2}$ & 30 & 100 & 0.68 \\
5 & $\mathbf{1 1 b}$ & 1.0 & $\mathrm{CD}_{2} \mathrm{Cl}_{2}$ & 30 & 70 & 0.65
\end{tabular}

${ }^{a}$ All reactions were carried out using $0.20 \mathrm{mmol}$ of 19 and $0.002 \mathrm{mmol}$ of catalyst in $0.8 \mathrm{ml}$ of solvent at $23^{\circ} \mathrm{C} .{ }^{b}$ Based on $19 .{ }^{c}$ Conversion of 19 to 20 determined by ${ }^{1} \mathrm{H}$ NMR analysis. ${ }^{d}$ Molar ratio of $E$ isomer and $Z$ isomer of 20 determined by ${ }^{1} \mathrm{H}$ NMR analysis.

\section{Catalyst syntheses}

General procedure for the synthesis of $\left\{\left[\mathrm{H}_{2} \mathrm{IMes}_{2}\right] \operatorname{RuCl}\left(\mathrm{PPh}_{3}\right)\right.$ $\left.\left[=\mathbf{C H}-\boldsymbol{o}-\left(\mathbf{O}^{\mathrm{i}} \mathbf{P r}\right) \mathbf{C}_{6} \mathbf{H}_{4}\right]\right\} \mathbf{X}(\mathbf{8})$. In a glove box, 7, the corresponding silver salt, triphenylphosphine and dichloromethane were added into a $20 \mathrm{ml}$ screw-cap vial equipped with a magnetic stir bar. The reaction mixture was stirred at room temperature for $2 \mathrm{~h}$ under dark. The resulting slurry was filtered and the filtrate was evaporated. The crude product was recrystallized from $\mathrm{CH}_{2} \mathrm{Cl}_{2}$-pentane or THF at $-20{ }^{\circ} \mathrm{C}$.

$\left\{\left[\mathbf{H}_{2} \mathrm{IMes}_{2}\right] \mathbf{R u C l}\left(\mathbf{P P h}_{3}\right)\left[=\mathbf{C H}-\boldsymbol{o}-\left(\mathbf{O}^{\mathrm{i}} \mathbf{P r}\right) \mathbf{C}_{6} \mathbf{H}_{4}\right]\right\} \mathbf{B F}_{4}$ (8a). Starting from 7 (300 mg, $479 \mu \mathrm{mol}), \mathrm{AgBF}_{4}(103 \mathrm{mg}, 527 \mu \mathrm{mol})$ and triphenylphosphine $(151 \mathrm{mg} 575 \mu \mathrm{mol})$ in $10 \mathrm{ml}$ of $\mathrm{CH}_{2} \mathrm{Cl}_{2}, \mathbf{8 a}$ was obtained as a red-orange crystalline solid $(397 \mathrm{mg}$, $422 \mu \mathrm{mol}, 88.1 \%$ yield based on 7$).{ }^{1} \mathrm{H}$ NMR $(500 \mathrm{MHz}$, $\mathrm{CD}_{2} \mathrm{Cl}_{2}$ ): $\delta / \mathrm{ppm} 15.16(\mathrm{~d}, J=7.0 \mathrm{~Hz}, 1 \mathrm{H}), 8.0-6.3(\mathrm{br}, 15 \mathrm{H})$, 7.55-7.51 (m, 1H), $7.24(\mathrm{~d}, J=8.5 \mathrm{~Hz}, 1 \mathrm{H}), 7.17$ (br s, 1H), 6.95-6.92 (m, 1H), 6.69-6.67 (m, 1H), 6.53 (br s, 1H), 6.42 (br s, 1H), 6.04 (br s, 1H), 5.74 (sep, $J=6.7 \mathrm{~Hz}, 1 \mathrm{H}), 4.20$ (br s, 1H), 4.05 (br s, 1H), 3.97 (br s, 1H), 3.63 (br s, 1H), 2.93 (br s, 3H), 2.35 (br s, 3H), 2.07 (br s, 3H), 1.97 (br s, 3H), 1.88-1.86 (br m, 6H), 1.59 (d, $J=6.7 \mathrm{~Hz}, 3 \mathrm{H}$ ), 1.30 (br s, 3H). ${ }^{13} \mathrm{C}\left\{{ }^{1} \mathrm{H}\right\}$ NMR (125.7 MHz, $\mathrm{CD}_{2} \mathrm{Cl}_{2}$ ): $\delta /$ ppm 289.4 (d), 208.9 (d), 154.1, 142.0, 141-140 (br m), 137-136 (br m), 136.0 (br s), 135.1 (br s), 135-134 (br m), 133-132 (br m), 132.7, 132-131 (br m), 130.0 (br s), 130-128 (br m), 126.9, 122.9, 118.0, 81.8, 23.4, 22.8, 22-21 (br, m), 20.7 (br s), 20-19 (br m), 17.7 (br s). ${ }^{31} \mathrm{P}\left\{{ }^{1} \mathrm{H}\right\}$ NMR (121.4 MHz, $\left.\mathrm{CD}_{2} \mathrm{Cl}_{2}\right): \delta / \mathrm{ppm}$ 53.6. HRMS (FAB+): Calculated for $\left\{\left[\mathrm{H}_{2} \mathrm{IMes}_{2}\right] \mathrm{RuCl}\left(\mathrm{PPh}_{3}\right)\left[=\mathrm{CH}-\mathrm{o}-\left(\mathrm{O}^{\mathrm{i}} \mathrm{Pr}\right) \mathrm{C}_{6} \mathrm{H}_{4}\right]\right\}^{+}$: 853.2628, Found: 853.2617.

$\left\{\left[\mathbf{H}_{2} \mathrm{IMes}_{2}\right] \mathbf{R u C l}\left(\mathrm{PPh}_{3}\right)\left[=\mathbf{C H}-\boldsymbol{o}-\left(\mathrm{O}^{\mathrm{i}} \mathrm{Pr}\right) \mathrm{C}_{6} \mathbf{H}_{4}\right]\right\} \mathrm{OTf} \quad$ (8b). Starting from 7 (300 mg, $479 \mu \mathrm{mol})$, AgOTf (135 mg, $527 \mu \mathrm{mol})$ and triphenylphosphine $(151 \mathrm{mg} 575 \mu \mathrm{mol})$ in $10 \mathrm{ml}$ of $\mathrm{CH}_{2} \mathrm{Cl}_{2}, \mathbf{8 b}$ was obtained as a red-orange crystalline solid $(277 \mathrm{mg}$, $276 \mu \mathrm{mol}, 57.6 \%$ yield based on 7$).{ }^{1} \mathrm{H}$ NMR $(500 \mathrm{MHz}$, $\mathrm{CD}_{2} \mathrm{Cl}_{2}$ ): $\delta / \mathrm{ppm} 15.15$ (d, $J=7.3 \mathrm{~Hz}, 1 \mathrm{H}$ ), 8.0-6.3 (br, 15H), $7.54-7.51(\mathrm{~m}, 1 \mathrm{H}), 7.22(\mathrm{~d}, J=8.2 \mathrm{~Hz}, 1 \mathrm{H}), 7.17$ (br s, 1H), 6.94-6.91 (m, 1H), 6.68-6.66 (m, 1H), 6.53 (br s, 1H), 6.42 (br s, 1H), 6.04 (br s, 1H), 5.74 (sep, $J=6.7 \mathrm{~Hz}, 1 \mathrm{H}), 4.19$ (br s, 1H), 4.04 (br s, 1H), 3.96 (br s, 1H), 3.64 (br s, 1H), 2.92 (br s, 3H), 2.35 (br s, 3H), 2.07 (br s, 3H), 1.97 (br s, 3H), 1.87-1.86 (br m, 6H), 1.58 (d, $J=6.7 \mathrm{~Hz}, 3 \mathrm{H}), 1.30$ (br s, 3H). ${ }^{13} \mathrm{C}\left\{{ }^{1} \mathrm{H}\right\}$ 
NMR (125.7 MHz, $\mathrm{CD}_{2} \mathrm{Cl}_{2}$ ): $\delta / \mathrm{ppm} 209.1$ (d), 154.2, 154.2, 142.0, 141-140 (br m), 137-136 (br m), 136.0 (br s), 135.1 (br s), 135-134 (br m), 133-132 (br m), 132.7, 132-131 (br m), 130.1 (br s), 130-128 (br m), 127.0, 122.9, 118.0, 81.8, 23.5, 22.8, 22-21 (br, m), 20.7 (br s), 20-19 (br m), 17.8 (br s). ${ }^{31} \mathrm{P}\left\{{ }^{1} \mathrm{H}\right\}$ NMR (121.4 MHz, $\left.\mathrm{CD}_{2} \mathrm{Cl}_{2}\right): \delta /$ ppm 53.5. HRMS (FAB+): Calculated for $\left\{\left[\mathrm{H}_{2} \mathrm{IMes}_{2}\right] \mathrm{RuCl}\left(\mathrm{PPh}_{3}\right)\left[=\mathrm{CH}-\mathrm{o}-\left(\mathrm{O}^{\mathrm{i}} \mathrm{Pr}\right) \mathrm{C}_{6} \mathrm{H}_{4}\right]\right\}^{+}$: 853.2628, Found: 853.2666.

$\left\{\left[\mathbf{H}_{2} \mathrm{IMes}_{2}\right] \mathbf{R u C l}\left(\mathbf{P P h}_{3}\right)\left[=\mathbf{C H}-\boldsymbol{o}-\left(\mathbf{O}^{\mathrm{i}} \mathbf{P r}\right) \mathbf{C}_{6} \mathbf{H}_{4}\right]\right\} \mathbf{P F}_{6}$ (8c). Starting from 7 (300 mg, $479 \mu \mathrm{mol}), \mathrm{AgPF}_{6}(133 \mathrm{mg}, 527 \mu \mathrm{mol})$ and triphenylphosphine $(151 \mathrm{mg} 575 \mu \mathrm{mol})$ in $10 \mathrm{ml}$ of $\mathrm{CH}_{2} \mathrm{Cl}_{2}, 8 \mathrm{c}$ was obtained as a red-orange crystalline solid (406 mg, $406 \mu \mathrm{mol}, 84.7 \%$ yield based on 7). ${ }^{1} \mathrm{H}$ NMR $(500 \mathrm{MHz}$, $\mathrm{CD}_{2} \mathrm{Cl}_{2}$ ): $\delta /$ ppm 15.16 (d, $\left.J=7.3 \mathrm{~Hz}, 1 \mathrm{H}\right), 8.0-6.3(\mathrm{br}, 15 \mathrm{H})$, $7.55-7.51(\mathrm{~m}, 1 \mathrm{H}), 7.23(\mathrm{~d}, J=8.5 \mathrm{~Hz}, 1 \mathrm{H}), 7.18$ (br s, $1 \mathrm{H})$, 6.95-6.92 (m, 1H), 6.69-6.68 (m, 1H), 6.53 (br s, 1H), 6.42 (br s, 1H), 6.05 (br s, 1H), 5.74 (sep, $J=6.7 \mathrm{~Hz}, 1 \mathrm{H}), 4.18$ (br s, 1H), 4.03 (br s, 1H), 3.96 (br s, 1H), 3.63 (br s, 1H), 2.93 (br s, 3H), 2.35 (br s, 3H), 2.07 (br s, 3H), 1.98 (br s, 3H), 1.88-1.86 (br m, 6H), 1.59 (d, $J=6.7 \mathrm{~Hz}, 3 \mathrm{H}$ ), 1.30 (br s, 3H). ${ }^{13} \mathrm{C}\left\{{ }^{1} \mathrm{H}\right\}$ NMR (125.7 MHz, $\mathrm{CD}_{2} \mathrm{Cl}_{2}$ ): $\delta /$ ppm 289.5 (d), 209.0 (d), 154.2, 154.2, 142.0, 141-140 (br m), 137-136 (br m), 136.0 (br s), 135.2 (br s), 135-134 (br m), 133-132 (br m), 132.7, 132-131 (br m), 130.0 (br s), 130-128 (br m), 127.0, 122.9, 118.0, 81.8, 23.5, 23.4, 22.8, 22-21 (br, m), 20.7 (br s), 20-19 (br m), 17.7 (br s). ${ }^{31} \mathrm{P}\left\{{ }^{1} \mathrm{H}\right\}$ NMR (121.4 MHz, $\mathrm{CD}_{2} \mathrm{Cl}_{2}$ ): $\delta / \mathrm{ppm}$ 53.5. HRMS $(\mathrm{FAB}+)$ : Calculated for $\left\{\left[\mathrm{H}_{2} \mathrm{IMes}_{2}\right] \mathrm{RuCl}\left(\mathrm{PPh}_{3}\right)\left[=\mathrm{CH}-\mathrm{o}-\left(\mathrm{O}^{\mathrm{i}} \mathrm{Pr}\right)\right.\right.$ $\left.\left.\mathrm{C}_{6} \mathrm{H}_{4}\right]\right\}^{+}:$853.2628, Found: 853.2647.

$\left\{\left[\mathrm{H}_{2} \mathrm{IMes}_{2}\right] \operatorname{RuCl}\left(\mathrm{PPh}_{3}\right)\left[=\mathrm{CH}-\boldsymbol{o}-\left(\mathrm{O}^{\mathrm{i}} \mathrm{Pr}\right) \mathrm{C}_{6} \mathrm{H}_{4}\right]\right\} \mathrm{SbF}_{6} \quad$ (8d). Starting from $7(300 \mathrm{mg}, 479 \mu \mathrm{mol}), \mathrm{AgSbF}_{6}(181 \mathrm{mg}, 527 \mu \mathrm{mol})$ and triphenylphosphine $(151 \mathrm{mg} 575 \mu \mathrm{mol})$ in $10 \mathrm{ml}$ of $\mathrm{CH}_{2} \mathrm{Cl}_{2}$, 8d was obtained as a red-orange crystalline solid (490 mg, $450 \mu \mathrm{mol}, 93.9 \%$ yield based on 7). ${ }^{1} \mathrm{H}$ NMR (500 MHz, $\mathrm{CD}_{2} \mathrm{Cl}_{2}$ ): $\delta /$ ppm 15.17 (d, $J=7.3 \mathrm{~Hz}, 1 \mathrm{H}$ ), 8.0-6.3 (br, 15H), 7.55-7.51 (m, 1H), 7.22 (d, J = 8.5 Hz, 1H), 7.18 (br s, 1H), 6.95-6.92 (m, 1H), 6.69-6.67 (m, 1H), 6.54 (br s, 1H), 6.42 (br s, 1H), 6.05 (br s, 1H), 5.74 (sep, $J=6.7 \mathrm{~Hz}, 1 \mathrm{H}), 4.16$ (br s, 1H), 4.02 (br s, 1H), 3.96 (br s, 1H), 3.62 (br s, 1H), 2.92 (br s, 3H), 2.35 (br s, 3H), 2.07 (br s, 3H), 1.98 (br s, 3H), 1.87-1.86 (br m, 6H), 1.59 (d, $J=6.7 \mathrm{~Hz}, 3 \mathrm{H}$ ), 1.31 (br s, 3H). ${ }^{13} \mathrm{C}\left\{{ }^{1} \mathrm{H}\right\}$ NMR (125.7 MHz, $\mathrm{CD}_{2} \mathrm{Cl}_{2}$ ): $\delta /$ ppm 289.5 (d), 209.1 (d), 154.2, 154.2, 142.0, 141-140 (br m), 137-136 (br m), 136.0 (br s), 135.2 (br s), 135-134 (br m), 133-132 (br m), 132.7, 132-131 (br m), 130.1 (br s), 130-128 (br m), 127.0, 122.9, 118.0, 81.8, 23.5, 23.4, 22.8, 22-21 (br, m), 20.7 (br s), 20-19 (br m), 17.7 (br s). ${ }^{31} \mathrm{P}\left\{{ }^{1} \mathrm{H}\right\}$ NMR (121.4 MHz, $\mathrm{CD}_{2} \mathrm{Cl}_{2}$ ): $\delta / \mathrm{ppm}$ 53.5. HRMS $(\mathrm{FAB}+)$ : Calculated for $\left\{\left[\mathrm{H}_{2} \mathrm{IMes}_{2}\right] \mathrm{RuCl}\left(\mathrm{PPh}_{3}\right)\left[=\mathrm{CH}-\mathrm{o}-\left(\mathrm{O}^{\mathrm{i}} \mathrm{Pr}\right) \mathrm{C}_{6} \mathrm{H}_{4}\right]\right\}^{+}$: 853.2628, Found: 853.2643.

General procedure for the synthesis of $\left\{\left[\mathrm{H}_{2} \mathrm{IMes}_{2}\right] \mathbf{R u C l}-\right.$ $\left.\left[\mathbf{P h}_{2} \mathbf{P}\left(\mathbf{C H}_{2}\right)_{n} \mathbf{P P h} \mathbf{h}_{2}\right](=\mathbf{C H P h})\right\}($ OTf $)$ (11). In a glove box, $\mathbf{9}$ and $\mathrm{CH}_{2} \mathrm{Cl}_{2}$ were added into a $20 \mathrm{ml}$ screw-cap vial equipped with a magnetic stir bar. With stirring, trimethylsilyl trifluoromethanesulfonate was added slowly. After stirring at room temperature for $1 \mathrm{~h}$, the corresponding diposphine was added. Then the reaction solution was stirred at room temperature for $2 \mathrm{~h}$, and evaporated. The crude product was dissolved in a small amount of $\mathrm{CH}_{2} \mathrm{Cl}_{2}$. The solution was added dropwise with vigorous stirring into a large amount of pentane (for 11a) or $\mathrm{Et}_{2} \mathrm{O}$ (for 11b). The appeared precipitate was corrected on a filter, washed with appropriate solvent, and dried under reduced pressure.

$\left\{\left[\mathrm{H}_{2} \mathrm{IMes}_{2}\right] \mathrm{RuCl}\left[\mathrm{Ph}_{2} \mathbf{P}\left(\mathrm{CH}_{2}\right)_{2} \mathbf{P P h}_{2}\right](=\mathrm{CHPh})\right\}(\mathrm{OTf})$ (11a). Starting from $9(100 \mathrm{mg}, 120 \mu \mathrm{mol})$, TMS(OTf) $(24.0 \mu \mathrm{l}$, $29.5 \mathrm{mg}, 133 \mu \mathrm{mol})$ and DPPE $(57.5 \mathrm{mg} 144 \mu \mathrm{mol})$ in $5.0 \mathrm{ml}$ of $\mathrm{CH}_{2} \mathrm{Cl}_{2}, 11 \mathrm{a}$ was obtained as a yellow-brown solid $(76.7 \mathrm{mg}$, $71.0 \mu \mathrm{mol}, 53.4 \%$ yield based on 9). ${ }^{1} \mathrm{H}$ NMR $(500 \mathrm{MHz}$, $\mathrm{CD}_{2} \mathrm{Cl}_{2}$ ): $\delta / \mathrm{ppm} 16.23(\mathrm{dd}, J=26.9 \mathrm{~Hz}, J=1.2 \mathrm{~Hz}, 1 \mathrm{H}$ ), 7.87-7.76 (m, 5H), 7.69-7.56 (m, 1H), 7.53-7.19 (m, 12H), 7.04 (s, 1H), 6.92-6.88 (m, 2H), 6.85-6.76 (m, 2H), 6.62-6.59 $(\mathrm{m}, 4 \mathrm{H}), 6.43(\mathrm{~s}, 1 \mathrm{H}), 5.60(\mathrm{~s}, 1 \mathrm{H}), 4.06-4.00(\mathrm{~m}, 1 \mathrm{H}), 3.93-3.87$ $(\mathrm{m}, 1 \mathrm{H}), 3.81-3.70(\mathrm{~m}, 2 \mathrm{H}), 2.76(\mathrm{~s}, 3 \mathrm{H}), 2.42(\mathrm{~s}, 3 \mathrm{H}), 2.28$ (s, 3H), 2.19-2.13 (br m, 1H), 2.10-2.03 (br m, 1H), 1.94 $(\mathrm{s}, 3 \mathrm{H}), 1.82(\mathrm{~s}, 3 \mathrm{H}), 1.40-1.34(\mathrm{br} \mathrm{m}, 1 \mathrm{H}), 1.17(\mathrm{~s}, 3 \mathrm{H})$, 0.68-0.64 (br m, 1H). ${ }^{13} \mathrm{C}\left\{{ }^{1} \mathrm{H}\right\}$ NMR (125.7 MHz, $\mathrm{CD}_{2} \mathrm{Cl}_{2}$ ): $\delta /$ ppm 213.1 (dd), 149.0, 141.5, 140.0, 139.6, 139.4, 138.3, $137.7,136.8,135.9$, 135.6, 135.3, 135.2, 135.0, 133.9, 133.9, 133.7, 133.3, 132.5-132.4 (m), 132.0, 131.9, 131.9, 131.3-131.2 (m), 130.9, 130.8-130.8 (m), 130.6-130.6 (m), 130.5, 130.4, 130.2-130.2 (m), 129.9, 129.0, 128.9, 128.9, 128.9, 128.5, 128.5, 128.4, 128.2, 41.4-41.0 (m), 21.8-21.4 (m), 21.0-20.9 (m), 20.6-20.5 (m), 18.9-18.9 (m), 18.0-18.0 (m), 15.9. ${ }^{31} \mathrm{P}\left\{{ }^{1} \mathrm{H}\right\} \mathrm{NMR}$ (121.4 MHz, $\mathrm{CD}_{2} \mathrm{Cl}_{2}$ ): $\delta / \mathrm{ppm} 53.4$ (d), 52.4 (d). HRMS (FAB+): Calculated for $\left\{\left[\mathrm{H}_{2} \mathrm{IMes}_{2}\right] \operatorname{RuCl}\left[\mathrm{Ph}_{2} \mathrm{P}\left(\mathrm{CH}_{2}\right)_{2} \mathrm{PPh}_{2}\right](=\mathrm{CHPh})\right\}^{+}$: 931.2651, Found: 931.2671.

$\left\{\left[\mathrm{H}_{2} \mathrm{IMes}_{2}\right] \mathrm{RuCl}\left[\mathrm{Ph}_{2} \mathbf{P}\left(\mathrm{CH}_{2}\right)_{3} \mathbf{P P h}_{2}\right](=\mathrm{CHPh})\right\}(\mathrm{OTf}) \quad(11 \mathrm{~b})$. Starting from $9(100 \mathrm{mg}, 120 \mu \mathrm{mol})$, TMS(OTf) $(24.0 \mu \mathrm{l}$, $29.5 \mathrm{mg}, 133 \mu \mathrm{mol})$ and DPPP (59.6 mg $144 \mu \mathrm{mol})$ in $5.0 \mathrm{ml}$ of $\mathrm{CH}_{2} \mathrm{Cl}_{2}, \mathbf{1 1 b}$ was obtained as a yellow solid $(78.7 \mathrm{mg}$, $71.9 \mu \mathrm{mol}, 59.7 \%$ yield based on 9). ${ }^{1} \mathrm{H} \mathrm{NMR}(500 \mathrm{MHz}$, $\mathrm{CD}_{2} \mathrm{Cl}_{2}$ ): $\delta / \mathrm{ppm} 16.83(\mathrm{dd}, J=26.5 \mathrm{~Hz}, J=1.2 \mathrm{~Hz}, 1 \mathrm{H}$ ), 7.68-7.60 (m, 7H), 7.50-7.44 (m, 3H), 7.39-7.36 (m, 2H), 7.32-7.28 (m, 3H), 7.17-7.14 (m, 3H), 7.11-7.08 (br m, 2H), 6.87-6.84 (m, 2H), 6.67-6.64 (br m, 2H), $6.62(\mathrm{~s}, 1 \mathrm{H}), 6.48$ (s, 1H), 6.41-6.37 (br m, 2H), $5.73(\mathrm{~s}, 1 \mathrm{H}), 4.02-3.95(\mathrm{~m}, 1 \mathrm{H})$, 3.84-3.78 (m, 1H), 3.71-3.66 (m, 2H), $2.83(\mathrm{~s}, 3 \mathrm{H}), 2.42(\mathrm{~s}, 3 \mathrm{H})$, 2.31-2.19 (br m, 3H), 2.12 (s, 3H), 1.95 (s, 3H), s1.89 (s, 3H), 1.78-1.73 (br m, 1H), 1.57-1.50 (br m, 1H), 1.15 (s, 3H), 0.93-0.80 (br m, 1H). ${ }^{13} \mathrm{C}\left\{{ }^{1} \mathrm{H}\right\}$ NMR (125.7 $\mathrm{MHz}, \mathrm{CD}_{2} \mathrm{Cl}_{2}$ ): $\delta /$ ppm 211.0 (dd), 150.2, 150.2, 141.5, 140.1, 139.4, 139.0, 138.3, 138.1, 137.5-137.5 (m), 137.2-137.2 (m), 137.1, 136.0, $135.9,135.6,134.7,134.7,133.7,133.5,133.4$, 133.2, 133.1, 131.7-131.7 (m), 131.6-131.6 (m), 131.3, 131.2, 131.0, 130.6, $130.6,130.2$, 130.1, 130.0, 129.8, 129.8, 129.4, 129.0, 128.7 . 128.5, 128.4, 127.6, 127.5, 36.5-36.2 (m), 23.4, 23.2, 21.5-21.0 (m), 19.4-19.4 (m), 18.3, 18.0-18.0 (m), 16.8. ${ }^{31} \mathrm{P}\left\{{ }^{1} \mathrm{H}\right\}$ NMR (121.4 MHz, $\mathrm{CD}_{2} \mathrm{Cl}_{2}$ ): $\delta /$ ppm 18.9 (d), 3.5 (d). HRMS (FAB+): Calculated for $\left\{\left[\mathrm{H}_{2} \mathrm{IMes}_{2}\right] \mathrm{RuCl}\left[\mathrm{Ph}_{2} \mathrm{P}\left(\mathrm{CH}_{2}\right)_{3} \mathrm{PPh}_{2}\right](=\mathrm{CHPh})\right\}^{+}$: 945.2808, Found: 945.2801.

\section{Crystal structure determination of $8 \mathrm{c}$}

Single crystals of $\mathbf{8 c}$ were recrystallised by diffusion of pentane into a saturated $\mathrm{CH}_{2} \mathrm{Cl}_{2}$ solution of $8 \mathrm{c}$; a suitable crystal was 
mounted on a hand-crafted glass fiber with Paratone oil (Exxon) and transferred to the $100 \mathrm{~K}$ cold gas stream of an OxfordCryosystems 700 Series Cryostream Cooler on the Bruker KAPPA APEXII 02B diffractometer.

Crystal data. $\mathrm{C}_{52} \mathrm{H}_{59} \mathrm{Cl}_{7} \mathrm{~F}_{6} \mathrm{~N}_{2} \mathrm{OP}_{2} \mathrm{Ru}, M=1253.17$, monoclinic, $a=10.1739(4), b=23.3587(10), c=23.0472(9) \AA ̊ ., U=5463.3$ (4) $\AA^{3}, T=100 \mathrm{~K}$, space group $P 2_{1} / n$ (no. 14), $Z=4,160797$ reflections measured, 21852 unique $\left(R_{\text {int }}=0.056\right)$, which were used in all calculations. Refinement of $F^{2}$ against all reflections (except the omitted $\left(\begin{array}{lll}0 & 1 & 1\end{array}\right)$ ) with the weights $\mathrm{w}=1 / \sigma^{2}\left(F_{\mathrm{o}}{ }^{2}\right)$. All non-hydrogen atoms were refined anisotropically. The cation and solvent hydrogen atoms were treated differently. All hydrogen atoms in the cation were freely refined with four parameters, three positional and one isotropic displacement. The six hydrogen atoms on the three dichloromethane molecules were included into the model at geometrically calculated positions and refined using a riding model. The isotropic displacement parameters of these hydrogen atoms were fixed to 1.2 times the $U_{\text {eq }}$ value of the carbon atoms to which they are bonded. The final $\mathrm{w} R\left(F^{2}\right)$ was 0.059 (all data).

\section{Metathesis assays}

Representative procedure for RCM of diethyldiallyl malonate (12). ${ }^{15}$ In a glove box, a $1.0 \mathrm{ml}$ volumetric flask was charged with $8 \mathrm{a}(7.5 \mathrm{mg}, 8.0 \mu \mathrm{mol})$ and $\mathrm{CD}_{2} \mathrm{Cl}_{2}$ was added to prepare $1.0 \mathrm{ml}$ of stock solution $(0.008 \mathrm{M}) \cdot \mathrm{CD}_{2} \mathrm{Cl}_{2}(700 \mu \mathrm{l})$ and the stock solution $(100 \mu \mathrm{l}, 0.80 \mu \mathrm{mol})$ were added into an NMR tube with a screw-cap septum top. The sample was equilibrated at $30^{\circ} \mathrm{C}$ in the NMR probe before $12(19.3 \mu \mathrm{l}, 19.2 \mathrm{mg}$, $80 \mu \mathrm{mol}$ ) was added via syringe. Data points were collected over an appropriate period of time using the Varian array function. The conversion of $\mathbf{1 2}$ to $\mathbf{1 3}$ was determined by comparing the ratio of the integrals of the methylene protons in the starting material with those in the product in the ${ }^{1} \mathrm{H}$ NMR spectra.

Representative procedure for CM of allylbenzene (14) and cis-1,4-diacetoxy-2-butene (15). ${ }^{\mathbf{1 5}}$

Preparation of a substrate mixture. In a glove box, tridecane (92.0 $\mu \mathrm{l}, 69.6 \mathrm{mg}, 377 \mu \mathrm{mol}), 14(100 \mu \mathrm{l}, 89.2 \mathrm{mg}, 755 \mu \mathrm{mol})$ and $15(240 \mu \mathrm{l}, 259 \mathrm{mg}, 1.51 \mathrm{mmol})$ were combined in a $5 \mathrm{ml}$ vial with a screw-cap septum top and a magnetic stir bar; this mixture was allowed to stir for $5 \mathrm{~min}$.

Preparation of reaction solution and $C M$ of 14 and 15 . In a glove box, a $5 \mathrm{ml}$ vial with a screw-cap septum top and a magnetic stir bar was charged with $8 \mathrm{a}(4.7 \mathrm{mg}, 5.0 \mu \mathrm{mol})$ and $\mathrm{CH}_{2} \mathrm{Cl}_{2}(1.0 \mathrm{ml})$. The catalyst solution was removed from the glove box and then stirred at $23{ }^{\circ} \mathrm{C}$ under argon. To the catalyst solution, the substrate mixture $(115 \mu \mathrm{l}$; tridecane: $24.5 \mu \mathrm{l}$, $18.5 \mathrm{mg}, 100 \mu \mathrm{mol}$; 14: $26.6 \mu \mathrm{l}, 23.7 \mathrm{mg}, 201 \mu \mathrm{mol}$; 15: $63.9 \mu \mathrm{l}$, $69.0 \mathrm{mg}, 401 \mu \mathrm{mol}$ ) was added via syringe. The reaction solution was allowed to stir at $23{ }^{\circ} \mathrm{C}$ and reaction aliquots $(c a .40 \mu \mathrm{l})$ were taken at the specific time points.

GC analysis. Samples for GC analysis were obtained by adding the reaction aliquot to $400 \mu \mathrm{l}$ of a $3 \mathrm{M}$ solution of ethyl vinyl ether in isopropyl alcohol. The sample was shaken and allowed to stand for $10 \mathrm{~min} .100 \mu \mathrm{l}$ of $1 \mathrm{M}$ slurry of tris (hydroxymethyl)phosphine in isopropyl alcohol was added. ${ }^{19}$
The sample was heated at $50{ }^{\circ} \mathrm{C}$ for $30 \mathrm{~min}$, cooled to room temperature, passed through a pad of silica gel using $\mathrm{CH}_{2} \mathrm{Cl}_{2}$ as eluent and then analyzed by GC. GC response factor and retention time for each substrate were summarized in Table S6. $\uparrow$ The amounts of the substrates in each sample were determined by previously reported method using response factors. ${ }^{15}$

Representative procedure for ROMP of norbornene (19). A $2 \mathrm{ml}$ volumetric flask was charged with 19 (125 mg, $1.33 \mathrm{mmol}$ ), and $\mathrm{CD}_{2} \mathrm{Cl}_{2}$ was added to prepare $2.0 \mathrm{ml}$ of stock solution $(0.665 \mathrm{M})$. In a glove box, 8a $(1.9 \mathrm{mg}, 2.0 \mu \mathrm{mol})$ and $\mathrm{CD}_{2} \mathrm{Cl}_{2}(500 \mu \mathrm{l})$ were added into an NMR tube with a screw-cap septum top. The stock solution (300 $\mu \mathrm{l}$; 19: $18.8 \mathrm{mg}, 200 \mu \mathrm{mol})$ was added via syringe and the sample was vigorously shaken for 30 seconds. Then the sample was allowed to stand for $30 \mathrm{~min}$ at $23{ }^{\circ} \mathrm{C}$ and analyzed by ${ }^{1} \mathrm{H}$ NMR. The conversion of 19 to 20 was determined by comparing the ratio of the integrals of the olefinic protons in the starting material with those in the product in the ${ }^{1} \mathrm{H}$ NMR spectrum.

\section{Conclusion}

While aiming to discover a convenient methodology for the preparation of cationic ruthenium alkylidene catalysts, simple ligand exchange using silver(I) salt of non-coordinating or weakly coordinating anion was investigated. When NHC-substituted catalyst 7 was reacted with a variety of silver(I) salt in the presence of $\mathrm{PPh}_{3}$, cationic catalysts bearing a single $\mathrm{PPh}_{3}$ ligand $\mathbf{8 a - d}$ were afforded selectively in good yield. Catalysts 8a-d were shown to exhibit moderate activity in the standard metathesis reactions. In contrast to reported neutral catalysts bearing a single bulky ligand, the stereoselectivities of 8a-d were very similar to that of dichloride substituted catalyst 7 . Additionally, diphosphines DPPE or DPPP were reacted with complex 9, causing formation of cationic diphosphine-chelated catalysts 11a-b. 11a-b exhibited poor activity in the standard RCM and CM compared to $\mathbf{8 a - d}$, most likely due to slow initiation derived from the high energy barrier of dechelation of the diphosphine ligand. Modification of the charge of ruthenium alkylidene catalyst is a promising avenue of investigation in improving catalyst efficiency and expanding their applications. The simple methods presented in this report will enable easy access to similar cationic catalysts and facilitate further investigations.

\section{Acknowledgements}

We thank Dr M. B. Herbert for helpful discussions and suggestions for this work, Materia, Inc. for the generous donation of catalysts and Dr M. W. Day and Mr L. M. Henling for X-ray crystallography. The Bruker KAPPA APEXII X-ray diffractometer was purchased via an NSF CRIF:MU award to the California Institute of Technology, CHE-0639094. This work was finan- 
cially supported by NIH (NIH 5R01GM031332), NSF (CHE-0410425 and CHE-0809418) and Mitsui Chemicals, Inc.

\section{References}

1 Selected reviews on olefin metathesis: (a) A. Fürstner, Angew. Chem., Int. Ed., 2000, 39, 3012; (b) T. M. Trnka and R. H. Grubbs, Acc. Chem. Res., 2001, 34, 18; (c) R. R. Schrock, Chem. Rev., 2002, 102, 145; (d) R. R. Schrock and A. H. Hoveyda, Angew. Chem., Int. Ed., 2003, 42, 4592; (e) C. Samojłowicz, M. Bieniek and K. Grela, Chem. Rev., 2009, 109, 3708.

2 J. Cossy, S. Arseniyadis and C. Meyer, Metathesis in Natural Product Synthesis: Strategies, Substrates, and Catalysts, Wiley-VCH, Weinheim, 1st edn, 2010.

3 J. B. Binder and R. T. Raines, Curr. Opin. Chem. Biol., 2008, 12, 767.

4 Y. Schrodi, T. Ung, A. Vargas, G. Mkrtumyan, C. W. Lee, T. M. Champagne, R. L. Pederson and S. H. Hong, Clean: Soil, Air, Water, 2008, 36, 669.

5 (a) A. Leitgeb, J. Wappel and C. Slugovc, Polymer, 2010, 51, 2927; (b) S. Sutthasupa, M. Shiotsuki and F. Sanda, Polym. J., 2010, 42, 905; (c) X. Liu and A. Basu, J. Organomet. Chem., 2006, 691, 5148.

6 G. C. Vougioukalakis and R. H. Grubbs, Chem. Rev., 2010, 110, 1746 and references cited therein.

7 (a) J. O. Krause, S. Lubbad, O. Nuyken and M. R. Buchmeiser, Adv. Synth. Catal., 2003, 345, 996; (b) J. O. Krause, S. H. Lubbad, O. Nuyken and M. R. Buchmeiser, Macromol. Rapid Commun., 2003, 24, 875; (c) J. O. Krause, O. Nuyken, K. Wurst and M. R. Buchmeiser, Chem. - Eur. J., 2004, 10, 777; (d) J. O. Krause, O. Nuyken and M. R. Buchmeiser, Chem. - Eur. J., 2004, 10, 2029.

8 (a) J. J. van Veldhuizen, S. B. Garber, J. S. Kingsbury and A. H. Hoveyda, J. Am. Chem. Soc., 2002, 124, 4954; (b) J. J. van Veldhuizen, D. G. Gillingham, S. B. Garber, O. Kataoka and A. H. Hoveyda, J. Am. Chem. Soc., 2003, 125, 12502; (c) D. G. Gillingham, O. Kataoka, S. B. Garber and A. H. Hoveyda, J. Am. Chem. Soc., 2004, 126, 12288; (d) J. J. van Veldhuizen, J. E. Campbell, R. E. Giudici and A. H. Hoveyda, J. Am. Chem. Soc., 2005, 127, 6877.

9 (a) K. Endo and R. H. Grubbs, J. Am. Chem. Soc., 2011, 133, 8525; (b) B. K. Keitz, K. Endo, P. R. Patel, M. B. Herbert and R. H. Grubbs, J. Am. Chem. Soc., 2012, 134, 693; (c) L. E. Rosebrugh, M. B. Herbert, V. M. Marx, B. K. Keitz and R. H. Grubbs, J. Am. Chem. Soc., 2013, 135, 1276; (d) K. Endo, M. B. Herbert and R. H. Grubbs, Organometallics, 2013, 32, 5128; (e) J. S. Cannon, L. Zou, P. Liu, Y. Lan, D. J. O'Leary, K. N. Houk and R. H. Grubbs, J. Am. Chem. Soc., 2014, 136, 6733; $(f)$ M. B. Herbert, B. A. Suslick, P. Liu, L. Zou, P. K. Dornan, K. N. Houk and R. H. Grubbs, Organometallics, 2015, 34, 2858.

10 (a) B. K. Keitz, K. Endo, M. B. Herbert and R. H. Grubbs, J. Am. Chem. Soc., 2011, 133, 9686; (b) B. K. Keitz,
A. Fedorov and R. H. Grubbs, J. Am. Chem. Soc., 2012, 134, 2040; (c) M. B. Herbert, V. M. Marx, R. L. Pederson and R. H. Grubbs, Angew. Chem., Int. Ed., 2013, 52, 310; (d) V. M. Marx, M. B. Herbert, B. K. Keitz and R. H. Grubbs, J. Am. Chem. Soc., 2013, 135, 94; (e) H. Miyazaki, M. B. Herbert, P. Liu, X. Dong, X. Xu, B. K. Keitz, T. Ung, G. Mkrtumyan, K. N. Houk and R. H. Grubbs, J. Am. Chem. Soc., 2013, 135, 5848; $(f)$ L. E. Rosebrugh, V. M. Marx, B. K. Keitz and R. H. Grubbs, J. Am. Chem. Soc., 2013, 135, 10032; $(g)$ J. Hartung and R. H. Grubbs, J. Am. Chem. Soc., 2013, 135, 10183; (h) J. S. Cannon and R. H. Grubbs, Angew. Chem., Int. Ed., 2013, 52, 9001; (i) J. Hartung and R. H. Grubbs, Angew. Chem., Int. Ed., 2014, 53, 3885; (j) S. L. Mangold, D. J. O'Leary and R. H. Grubbs, J. Am. Chem. Soc., 2014, 136, 12469; (k) J. Hartung, P. K. Dornan and R. H. Grubbs, J. Am. Chem. Soc., 2014, 136, 13029; (l) M. B. Herbert and R. H. Grubbs, Angew. Chem., Int. Ed., 2015, 54, 5018; (m) P. K. Dornan, Z. K. Wickens and R. H. Grubbs, Angew. Chem., Int. Ed., 2015, 54, 7134.

11 (a) N. Audic, H. Clavier, M. Mauduit and J.-C. Guillemin, J. Am. Chem. Soc., 2003, 125, 9248; (b) H. Clavier, N. Audic, M. Mauduit and J.-C. Guillemin, Chem. Commun., 2004, 2282; (c) H. Clavier, N. Audic, J.-C. Guillemin and M. Mauduit, J. Organomet. Chem., 2005, 690, 3585; (d) Q. Yao and Y. Zhang, Angew. Chem., Int. Ed., 2003, 42, 3395; (e) Q. Yao and M. Sheets, J. Organomet. Chem., 2005, 690, 3577.

12 (a) M. A. O. Volland, S. M. Hansen, F. Rominger and P. Hofmann, Organometallics, 2004, 23, 800; (b) M. Zirngast, E. Pump, A. Leitgeb, J. H. Albering and C. Slugovc, Chem. Commun., 2011, 47, 2261; (c) O. Songis, A. M. Z. Slawin and C. S. J. Cazin, Chem. Commun., 2012, 48, 1266; (d) B. Autenrieth, W. Frey and M. R. Buchmeiser, Chem. Eur. J., 2012, 18, 14069; (e) B. Autenrieth, E. B. Anderson, D. Wang and M. R. Buchmeiser, Macromol. Chem. Phys., 2013, 214, 33; $(f)$ A. M. McKinty, C. Lund and D. W. Stephan, Organometallics, 2013, 32, 4730.

13 S. B. Garber, J. S. Kingsbury, B. L. Gray and A. H. Hoveyda, J. Am. Chem. Soc., 2000, 122, 8168.

14 M. S. Sanford, J. A. Love and R. H. Grubbs, J. Am. Chem. Soc., 2001, 123, 6543.

15 T. Ritter, A. Hejl, A. G. Wenzel, T. W. Funk and R. H. Grubbs, Organometallics, 2006, 25, 5740.

16 (a) P. Teo and R. H. Grubbs, Organometallics, 2010, 29, 6045; (b) G. Occhipinti, F. R. Hansen, K. W. Törnroos and V. R. Jensen, J. Am. Chem. Soc., 2013, 135, 3331; (c) V. R. Jensen, G. Occhipinti and F. R. Hansen, Int. Patent Appl, WO2012/032131A1, 2012.

17 Conversion of $\mathbf{1 4}$ to 16 slightly decreased between $240 \mathrm{~min}$ and $480 \mathrm{~min}$. We speculate that this was caused by secondary metathesis involving $\mathbf{1 6}$ as a reactant. Similar phenomenon has been observed in other metathesis reactions by ruthenium alkylidene catalysts. See ref. 15 for the data.

18 A. B. Pangborn, M. A. Giardello, R. H. Grubbs, R. K. Rosen and F. J. Timmers, Organometallics, 1996, 15, 1518.

19 R. L. Pederson, I. M. Fellows, T. A. Ung, H. Ishihara and S. P. Hajela, Adv. Synth. Catal., 2002, 344, 728-735. 